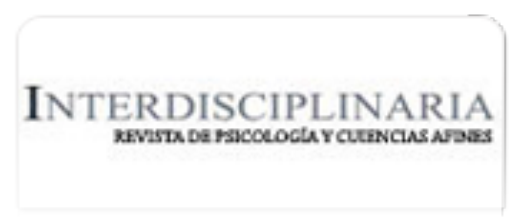

Interdisciplinaria

ISSN: 0325-8203

ISSN: 1668-7027

interdisciplinaria@fibercorp.com.ar

Centro Interamericano de Investigaciones Psicológicas y

Ciencias Afines

Argentina

Palomar Lever, Joaquina; Victorio Estrada, Amparo

Predictores y correlatos del comportamiento prosocial de adolescentes mexicanos

Interdisciplinaria, vol. 35, núm. 2, 2018, Julio-, pp. 495-509

Centro Interamericano de Investigaciones Psicológicas y Ciencias Afines

Argentina

Disponible en: https://www.redalyc.org/articulo.oa?id=18058785015

Cómo citar el artículo

Número completo

Más información del artículo

Página de la revista en redalyc.org

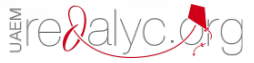

Sistema de Información Científica Redalyc

Red de Revistas Científicas de América Latina y el Caribe, España y Portugal

Proyecto académico sin fines de lucro, desarrollado bajo la iniciativa de acceso

abierto 


\title{
Predictores y correlatos del comportamiento prosocial de adolescentes mexicanos*
}

\section{Predictors and correlates of prosocial behavior of Mexican adolescents}

\author{
Joaquina Palomar Lever** y Amparo Victorio Estrada***
}

\begin{abstract}
*Investigación financiada por el fondo mixto Conacyt-Sedesol No. 91388, concedido a la primera autora. **Doctora en Psicología. Profesora investigadora. Departamento de Psicología de la Universidad Iberoamericana, Ciudad de México.

***Doctora en Ciencias Naturales. Asistente de investigación. Departamento de Psicología de la Universidad Iberoamericana, Ciudad de México. E-mail: victorioconsulting@gmail.com
\end{abstract}

Universidad Iberoamericana. Ciudad de México, México.

\section{Resumen}

La conducta prosocial comprende comportamientos altruistas en favor de otras personas y conlleva un beneficio social mayor como la cooperación y el apoyo y cuidado de los demás. El comportamiento prosocial resulta también en un beneficio personal al tener éste un efecto social positivo para quien lo realiza, asociado a resultados significativos para el ajuste psicosocial de los jóvenes. El objetivo de este estudio fue identificar predictores de la conducta prosocial en adolescentes mexicanos que viven en condiciones de pobreza. Se analizaron los datos de 1093 adolescentes, provenientes de una muestra nacional probabilística de hogares de localidades rurales y urbanas que están inscritos en un programa gubernamental de combate a la pobreza $(55.8 \%$ hombres, $61 \%$ urbanos, edad promedio 14.92 años y escolaridad promedio 8.33 años cursados). No se encontraron diferencias en conducta prosocial por tipo de localidad; pero sí por género en favor de las mujeres, aunque el tamaño del efecto fue pequeño. El modelamiento de ecuaciones estructurales mostró que niveles más elevados de conducta prosocial dependen de mayor competencia social, relaciones positivas con los pares, apoyo social general y de amigos, estilo parental permisivo y soledad; así como de menor oposicionismo y menor cantidad de menores de 12 años en el hogar. El modelo explicó el $60 \%$ de la varianza de la conducta prosocial en los adolescentes. Los mejores predictores de la conducta prosocial fueron el comportamiento social y el apoyo percibido: Quienes son socialmente más eficientes, exitosos y más apoyados, tienden a retribuir con mayor altruismo hacia sus pares.

Palabras clave: Conducta prosocial; Habilidades sociales; Adolescentes; Pobreza, México.

\begin{abstract}
Prosocial behavior refers to altruistic behavior in favor of other people. Prosocial behavior also results in a personal benefit for those who perform it, because it has a positive social effect and plays a transcendental role in the development of young people and their psychosocial adjustment. The present study analyses some social behaviors of adolescents living in poverty, with the aims of determining the predictors of their prosocial behavior and possible differences in prosocial behavior by gender and by type of place of residence. Two hypotheses
\end{abstract}


were tested: that there are gender differences in the prosocial behavior of adolescents and that the social behavior of adolescents predicts their tendency towards prosocial behavior. No hypothesis was established for the type of locality. In this survey participated 1093 adolescents, 55.8\% men, $61 \%$ from urban localities, average age of $14.92(S D=1.29)$ years, and average schooling $8.33(S D=1.71)$ years. Access to the national registry of the government's poverty reduction program was attained, from which a probabilistic selection of registered households was obtained, with national representativeness for the rural and urban domains. Households with adolescent children were selected from the national sample of households, to visit them in a second survey to interview the children of the heads of these households. Analyses were conducted to determine differences in prosocial behavior by gender and urbanization. Results showed that male and female scores differed significantly, in favor of females. However, the prosocial behavior scores of rural and urban youths did not differ significantly. To identify potential predictors of prosocial behavior, a structural equation modeling was performed. The model included a latent variable called Socialization, which was made up of scales that measure social competence, positive relationships with peers, social support and support from friends. Permissive parental style, oppositional behavior, loneliness, religiosity, and the number of children under 12 in the household were also tested. The resulting model showed that Socialization was the main predictor of the prosocial behavior of adolescents. Permissive parental style and loneliness were also positive predictors of prosocial behavior; while oppositional behavior and the number of children under the age of 12 in the household predicted it negatively. The tested model reached an adequate fit and explained $61 \%$ of the total variance of prosocial behavior. The present data supported the hypotheses of the study since we observed gender differences in the prosocial behavior of adolescents; and the structural equations modeling confirmed the relative importance of indicators of social behavior, grouped into Socialization, in the prediction of prosocial behavior. However, prosocial behavior did not differ by type of residence. The findings confirmed the close relationship between interpersonal relationships, social support, and prosocial behavior; since those who were socially more efficient and successful, and received more support tended to reciprocate with greater altruism towards their peers. Additionally, less oppositional behavior was associated with greater altruism. The positive relationship between loneliness and prosocial behavior indicated that the tendency towards social isolation promotes altruistic behavior. Results also suggested that less disciplinary parental control promotes behavior in favor of others; but not the coexistence with young children. No direct relationship between religiosity and prosocial behavior was found. Among the strengths of this study should be mentioned that the results obtained came from a national probabilistic sample of households and the large size of the sample; as well as some caveats like the cross-sectional design of the survey and the use of self-reports. However, we consider that it is possible to use the present results in the planning of intervention strategies with adolescents, with the purpose of stimulating prosocial behavior in future generations.

Key words: Prosocial behavior; Social skills; Adolescents; Poverty; Mexico.

\section{Introducción}

La conducta prosocial se refiere a comportamientos altruistas en favor de otras personas como confortar y ayudar a los demás, así como compartir y donar a los más necesitados. El estudio de la conducta prosocial es relevante ya que ésta forma parte de la convivencia cotidiana entre las diversas personas de una sociedad, facilitando la cohesión del grupo social. Adicionalmente, la conducta prosocial puede coadyuvar a construir un ambiente social más armonioso por ser opuesta al comportamiento agresivo y contribuir al progreso general de una sociedad al apoyar a los más vulnerables del grupo social. 


\section{Características del comportamiento prosocial}

Dentro de las características que definen a la conducta prosocial se incluye la de tratarse de un comportamiento voluntario, con la intención de beneficiar a otras personas, sin importar si dicho comportamiento le reporta un costo o un beneficio a quien lo realiza (Allgaier, Zettler, Wagner, Püttmann $\&$ Trautwein, 2015). La conducta prosocial es relevante por sí misma porque conlleva un beneficio social mayor, como la cooperación, el apoyo y el cuidado de los demás, y porque, al ser altruista, es opuesta al comportamiento agresivo, lo que en conjunto puede ser considerado como crucial para la armonía social (Lai, Siu, \& Shek, 2015). Si se considera que la conducta prosocial está dirigida al beneficio de otras personas, este comportamiento puede ser descrito como positivo, deseable y meritorio de auspicio.

En este sentido, la conducta altruista por sí misma tiene una connotación positiva y existen datos que señalan que el comportamiento prosocial de los adolescentes está relacionado con características positivas de quienes lo realizan (Ma, Cheung, \& Shek, 2007). Sin embargo, otros aspectos menos positivos relacionados con la conducta prosocial han recibido atención en la literatura. Uno de estos aspectos es que la conducta prosocial puede ser puramente altruista, así como fungir como un mecanismo de validación social (Eberly-Lewis \& Coetzee1, 2015). La literatura ha destacado la posible relación entre la conducta prosocial y ciertos rasgos como el narcisismo y el comportamiento manipulativo. Esto se debe a que la conducta prosocial se relaciona positivamente con una visión positiva de sí mismo y que una percepción positiva de la propia competencia social se relaciona positivamente con el narcisismo (Kauten \& Barry, 2016). En este sentido, se ha argumentado que los jóvenes pueden aprovechar las situaciones prosociales como una oportunidad para el enaltecimiento de sí mismos y obtener validación de su propia percepción de superioridad sobre los de- más (Eberly-Lewis \& Coetzee1, 2015), o utilizar la conducta prosocial como una estrategia manipulativa para congraciarse con otros y ganar su favor (Kauten \& Barry, 2016).

Otros aspectos relevantes en esta discusión son la deseabilidad social y la inteligencia emocional. La primera consiste precisamente en el manejo de la impresión para obtener el reconocimiento de los demás; mientras que la segunda permite a los individuos identificar las necesidades de los otros y cómo satisfacerlas. En ambos casos se trata de comportamientos que podrían servir al propósito de manipular la percepción de los demás en beneficio propio y obtener así admiración y alabaza (Kauten \& Barry, 2016), que conceptualiza el autosacrificio inherente al altruismo como una estrategia de autoenaltecimiento.

Para aclarar esta relación es conveniente diferenciar entre la conducta prosocial altruista $\sin$ beneficio propio (la ayuda de forma anónima) y la conducta prosocial pública, de la que la persona puede obtener una ventaja como el reconocimiento social. Al respecto, los resultados obtenidos por Eberly-Lewis y Coetzeel (2015) sugieren que la empatía (la habilidad de ponerse en la situación de otros y de expresar compasión y preocupación por la persona infortunada) se relaciona positivamente con la tendencia a beneficiar a otros y negativamente con la tendencia a beneficiarse a sí mismos; mientras, el narcisismo se relaciona positivamente con tendencias prosociales que conllevan un beneficio propio. Los resultados de los citados autores también mostraron que, tanto los rasgos egoístas como la preocupación acerca de la aprobación de los demás, predicen la conducta prosocial pública y oportunista; mientras que la empatía predice la conducta prosocial anónima, sin esperar recompensa.

Así también, niveles más altos de conducta prosocial, independientemente de la obtención de un beneficio propio, están asociados con altos niveles del rasgo honestidad-humildad; mientras que niveles bajos de este rasgo se asocian con conducta pro- 
social ligada a la obtención de un beneficio y a conducta antisocial (e.g., explotación) relacionada con la posibilidad de impunidad (Allgaier et al., 2015). Otros resultados añaden más información sobre esta diferenciación entre altruismo interesado y desinteresado, y revelan que la deseabilidad social no está asociada con la conducta prosocial autoreportada o reportada por terceros, ni modera la relación entre narcisismo y conducta prosocial (Barry, Lui, \& Anderson, 2017). En este sentido, la conducta prosocial puede ser considerada como un comportamiento válido en sí mismo y no como un artificio debido a la deseabilidad social (JensenCampbell, Knack, \& Gomez, 2010).

En relación a la evolución de la conducta prosocial, la investigación previa revela que mientras algunos resultados han documentado que la conducta prosocial se mantiene estable desde la mitad de la niñez hasta la adolescencia tardía (Flynn, Ehrenreich, Beron, \& Underwood, 2015); otros estudios señalan que la conducta prosocial disminuye en la adolescencia temprana para después aumentar en la adolescencia tardía (Carlo, Padilla-Walker, \& Nielson, 2015; Lai et al., 2015; Malti et al., 2015; WrayLake, Syvertsen, \& Flanagan, 2016). Adicionalmente se ha encontrado que a nivel intraindividual se observa durante la adolescencia el decremento y aumento subsecuente en los niveles de conducta prosocial previamente mencionados; mientras que a nivel interindividual las diferencias personales en conducta prosocial permanecen relativamente estables durante dicho periodo (Carlo, Crockett, Randall, \& Roesch, 2007). Es decir, la conducta prosocial fluctúa intraindividualmente, pero las diferencias interindividuales se mantienen.

Respecto a las diferencias de género en la conducta prosocial, la literatura ha mostrado resultados divergentes. Algunos datos muestran diferencias en conducta prosocial en favor de las mujeres (Brittian \& Humphries, 2015); mientras que otros resultados muestran niveles equiparables de conducta prosocial entre hombres y mujeres (Guevara, Cabrera, Gonzalez, \& Devis, 2015) y niveles más altos en los hombres que en las mujeres (Lai et al., 2015). También se han determinado diferencias culturales consistentes en una mayor tendencia a manifestar conducta prosocial hacia quienes comparten el mismo trasfondo cultural (Brittian \& Humphries, 2015).

\section{Predictores del comportamiento prosocial}

La mencionada disminución y aumento subsecuente entre la mitad y final de la adolescencia en los niveles de conducta prosocial concuerda con la evolución de otros procesos que pueden estar relacionados con ésta. Por ejemplo, coincide con que los valores de responsabilidad social disminuyen entre la niñez tardía y la adolescencia intermedia, para luego aumentar y estabilizarse en la adolescencia tardía (Wray-Lake et al., 2016). Asímismo, la evolución de la conducta prosocial coincide con que el razonamiento moral se vuelve más complejo y sofisticado en el transcurso de la niñez a la adolescencia, hasta alcanzar una orientación empática (i.e., expresión de preocupación compasiva por los otros) en la adolescencia tardía y adultez temprana (Lai et al., 2015). Sin embargo, los factores familiares y escolares de influencia social (e.g., padres, pares) son predictores más fuertes que los factores de competencia individual como la empatía y razonamiento moral (Lai et al., 2015).

El mencionado aumento en la conducta social en la adolescencia tardía coincide también con el aumento en la importancia de la influencia de los pares en el comportamiento de los adolescentes, la cual es importante porque la socialización y el fomento de normas y conductas altruistas influyen en el comportamiento prosocial de los jóvenes (Lai et al., 2015). En este sentido, los pares afectan la conducta prosocial de los adolescentes, tanto positiva como negativamente, a través de procesos de reforzamiento social, por lo que la influencia de los pares juega un rol trascendental en la transmisión de normas sociales del grupo e influye en el desarrollo prosocial y en el ajuste social de los adolescentes (van Ho- 
orn, van Dijk, Meuwese, Rieffe, \& Crone, 2016). Esta influencia se refleja en la asociación positiva de la conducta prosocial con la interacción e influencia positivas de los pares y en la asociación negativa del comportamiento prosocial con la influencia negativa y la conducta delincuente de aquellos (Ma et al., 2007).

Tanto la popularidad (el grado con el que son admirados por sus pares) como la aceptación (el grado con el que son apreciados por sus pares) se asocian positivamente con la conducta prosocial en los adolescentes (Closson \& Hymel, 2016; Laninga-Wijnen, Harakeh, Dijkstra, Veenstra, \& Vollebergh, 2016) y en los varones y mujeres entre la adolescencia tardía y la adultez temprana (Ruschoff, Dijkstraa, Veenstra, \& Lindenberg, 2015). La conducta prosocial funge también como factor protector contra la victimización por acoso, ya que promueve la vinculación con los pares y contrarresta el rechazo por parte de éstos, tanto en mujeres como en varones (Wang et al., 2015).

Otro aspecto relacionado con la conducta prosocial es la percepción de apoyo social por parte de los pares, ya que los jóvenes que experimentan mayor apoyo social muestran también más conducta prosocial (Plenty, Östberg, \& Modin, 2015). Esta asociación puede estar relacionada con que en el periodo entre la mitad y el final de la adolescencia se desarrolla un sentido de reciprocidad respecto a los vínculos sociales, de tal manera que las decisiones de los jóvenes respecto a la inversión de recursos personales en beneficio de sus pares está determinada por sus sentimientos recíprocos de amistad (Burnett Heyes et al., 2015). Al respecto, los resultados obtenidos por van Rijsewijk, Dijkstra, Pattiselanno, Steglich y Veenstra (2016) documentan una falta de reciprocidad en el intercambio de ayuda entre pares a favor de los adolescentes más rechazados y con menor rendimiento académico. Esto sugiere la posibilidad de que la compasión juegue un papel importante en la relación positiva entre la vinculación con los pares y la conducta prosocial (PadillaWalker, Fraser, Black, \& Bean, 2015).

Una fuente de influencia social relacio- nada con el altruismo y la compasión es la religiosidad, debido a que estos valores con frecuencia son avalados por los grupos religiosos. La literatura documenta la relación positiva entre religiosidad y conducta prosocial tanto auto-reportada (Brittian \& Humphries, 2015; Li \& Chow, 2015), como reportada por terceros (Saroglou, Pichon, Trompette, Verschueren, \& Dernelle, 2005). Estos autores sugieren que la conducta prosocial de las personas con altos niveles de religiosidad, no es producto de un artificio debido a la deseabilidad social, ni a otros factores como el género, el apego, la empatía o la honestidad, sino que se trata de una relación genuina (Saroglou et al., 2005). Sin embargo, esta relación es compleja, ya que los resultados de Li y Chow (2015) muestran que la relación entre religiosidad y conducta prosocial es curvilínea (niveles más altos y más bajos de religiosidad reportan mayor conducta prosocial, pero no los niveles medios), lo que sugiere la necesidad de mayor investigación para esclarecer este punto.

Los padres como agentes de socialización tienen un efecto importante en la conducta prosocial de sus hijos que se ve reflejado en que un buen ambiente social familiar aumenta el comportamiento prosocial (Ma et al., 2007) y que el apoyo parental influye positivamente en la conducta prosocial hacia los familiares y pares (Mesurado \& Richaud, 2016). En contraste, las prácticas parentales de control psicológico (Padilla-Walker et al., 2015), así como los estilos maternos inconsistentes de disciplina (Chaparro \& Grusec, 2016) se relacionaron negativamente con la conducta prosocial de los adolescentes. Un aspecto importante es que se ha observado que la relación entre las prácticas parentales y la conducta prosocial de los adolescentes es bidireccional; ésto significa que la conducta prosocial de los adolescentes influye en las prácticas parentales subsecuentes y viceversa (PadillaWalker, Carlo, Christensen, \& Yorgason, 2012). Así también, niveles más altos de conducta prosocial en la niñez tardía aumentan la calidad de la relación entre ma- 
dres e hijos a mediano plazo, resultado que ha sido observado en diferentes países con una extensa variedad de condiciones sociodemográficas y características psicológicas (Pastorelli et al., 2016). Dentro de los aspectos familiares que también pueden influir en el comportamiento prosocial se ha estudiado la relación fraternal que existe en el hogar. En cuanto a la relevancia de un ambiente social positivo, se ha encontrado que el afecto fraternal entre hermanos está positivamente asociado con el comportamiento futuro de compasión y conducta prosocial de los adolescentes (Harper, PadillaWalker, \& Jensen, 2016).

\section{Presente estudio}

El comportamiento prosocial es intrínsecamente positivo, ya que la característica que lo define es que está dirigido al beneficio de otras personas y, por ello, es por sí mismo deseable. Sin embargo, la conducta prosocial resulta también en un beneficio personal al tener ésta un efecto social positivo para quien la realiza. Dentro de estos efectos benéficos cabe señalar relaciones interpersonales más satisfactorias, mayor competencia social y mayor bienestar personal (Jensen-Campbell et al., 2010; Lai et al., 2015), mejor calidad en la relación entre madres e hijos (Pastorelli et al., 2016) y, además, reducir el rechazo de los pares, promover la vinculación con éstos y disminuir la victimización relacional por parte de los pares (Wang et al., 2015). Además, la conducta prosocial está asociada a varios aspectos positivos como el éxito académico y la satisfacción con el logro personal (Lai et al., 2015), así como menor consumo de sustancias (Carlo, Crockett, Wilkinson, \& Beal, 2011), menos síntomas externalizantes en varones y menos síntomas internalizantes en mujeres (Flynn et al., 2015). En este sentido, la conducta prosocial puede ser considerada como instrumental en la consecución de relaciones interpersonales duraderas, felicidad, éxito y bienestar (Jensen-Campbell et al., 2010). De tal manera que, independientemente de su valor social general, el comportamiento prosocial juega un papel trascendental en el desarrollo de los jóvenes, porque está asociado a resultados significativos para su ajuste psicosocial y se ubica por ello dentro del denominado desarrollo positivo de los adolescentes (Lai et al., 2015; Ruschoff et al., 2015).

Como futuros adultos, los adolescentes forman un grupo de interés, ya que en ellos reside la posibilidad de crear un ambiente social ulterior más solidario y armonioso. Esta posibilidad hace relevante estudiar los predictores de la conducta prosocial en una época de la vida, en la que el comportamiento puede ser más susceptible al cambio y más responsivo a estrategias de influencia social. Por otra parte, la conducta prosocial adquiere mayor relevancia en contextos de pobreza extrema, en los que las necesidades superan por mucho los recursos económicos y en los que la ayuda disponible usualmente consiste en el apoyo de otros en términos de tiempo y labor, más que en términos pecuniarios.

La literatura revisada sugiere que la promoción de la conducta prosocial en los adolescentes es una meta socialmente deseable, por lo que estudiar sus determinantes es importante para identificar los factores que deben ser considerados en el planteamiento de intervenciones psicosociales que la promuevan. A partir de los resultados revisados con antelación, el comportamiento social de los adolescentes, en términos de su interacción con otras personas, se perfila como un factor importante en la determinación de los niveles de conducta prosocial. Así también, otros factores como los estilos de crianza y la religiosidad merecen ser considerados.

El presente estudio aborda el análisis de dichos factores con el objetivo de determinar qué elementos pueden predecir la conducta prosocial en adolescentes que viven en condiciones de pobreza. Adicionalmente, este estudio analiza posibles diferencias de género y por tipo de localidad de residencia respecto a los niveles de comportamiento prosocial reportado. Se sometieron a prueba dos hipótesis: la primera estipula que existen diferencias de género en el comportamiento prosocial de los adolescentes y la segunda establece que el comportamiento 
social de los adolescentes predice su tendencia hacia la conducta prosocial. No se estableció una hipótesis para el tipo de localidad, debido a la carencia de información respectiva en la literatura.

\section{Método}

\section{Participantes}

Hijos. En esta encuesta participaron 1093 adolescentes, $55.8 \%$ hombres, con una edad promedio de 14.92 años $(D E=1.29)$ y 8.33 años $(D E=1.71)$, de escolaridad promedio. El $61 \%$ de los adolescentes provinieron de localidades urbanas.

Muestreo. Se obtuvo acceso al padrón nacional del programa gubernamental de combate a la pobreza, del cual se obtuvo una selección probabilística de los hogares registrados. Se seleccionó la muestra de hogares para los dominios rural y urbano por separado a través de un muestreo bietápico: en la primera etapa se seleccionaron las localidades mediante probabilidad proporcional al tamaño y en la segunda etapa se seleccionaron los hogares dentro de las localidades por muestreo aleatorio simple. La selección excluyó a los hogares sin datos económicos y provenientes de localidades indígenas (no hispanoparlantes) y menores de 45 hogares. Este procedimiento resultó en una muestra de 2012 hogares encuestados, con representatividad nacional para los dominios rural y urbano. De esta muestra nacional de hogares se seleccionaron los hogares con hijos adolescentes, para visitarlos en una segunda encuesta y entrevistar a los hijos de los titulares de estos hogares. De esta segunda encuesta proceden los datos de los 1093 adolescentes aquí analizados.

\section{Instrumentos}

Todas las escalas utilizadas en este estudio fueron validadas previamente en adolescentes mexicanos (Palomar, 2015).

Conducta prosocial. Mide la tendencia hacia la empatía y el altruismo orientado hacia los demás. La escala consta de nueve ítems, que proceden de otros instrumentos (Caprara \& Pastorelli, 1993; Davis, 1980) (confiabilidad $\alpha=.88$, rango: $1-4, M=3.02$, $D E=.63)$.

Competencia social. Mide la percepción de capacidad y aptitud en situaciones de interacción social en el ámbito escolar. La escala consta de cinco ítems procedentes de otro instrumento (Gresham \& Elliot, 1990) (confiabilidad $\alpha=.82$, rango: $1-4, M=$ 3.21, $D E=.59)$.

Relación con pares. Mide el grado en el que la persona se percibe como sociable y popular entre sus pares; consta de siete ítems provenientes de otro instrumento (DuBois, Felner, Brand, Phillips, \& Lease, 1996) (confiabilidad $\alpha=.83$, rango: $1-4, M=3.12, D E=$ $.60)$.

Apoyo. Los ítems proceden de otro instrumento (Zimet, Dahlem, Zimet, \& Farley, 1998) y están agrupados en dos subescalas: Apoyo social (12 ítems, confiabilidad $\alpha=.93$, rango: $1-4, M=3.28, D E=.64)$ y Apoyo de amigos (tres ítems, confiabilidad $\alpha=.77$, rango: $1-4, M=2.98, D E=.77)$. Las subescalas miden la percepción de disponibilidad de personas que aportan apoyo y comprensión en general y por parte del círculo de amistades, respectivamente.

Soledad. Los ítems provienen de otro instrumento (de Jong Gierveld \& van Tilburg, 1999). La escala mide la tendencia al aislamiento social y consta de tres ítems (confiabilidad $\alpha=.57$, rango: $1-4, M=1.66$, $D E=.63$ ).

Oposicionismo. Mide la tendencia al comportamiento antagónico y retador, en especial contra los adultos. La escala consta de ocho ítems provenientes de otro instrumento (Pelham, Gnagy, Greenslade, \& Milich, 1992) (confiabilidad $\alpha=.85$, rango: $1-4$, $M=1.51, D E=.51)$.

Religiosidad. Mide la tendencia a tener convicciones religiosas fuertes y un mayor compromiso con los preceptos religiosos. La escala consta de 27 ítems (confiabilidad $\alpha=.95$, rango: $1-4, M=3.06, D E=.58)$. Los ítems provienen de otros instrumentos 
(Grulke et al., 2003; Koenig, Pargament, Perez, \& Smith, 1998).

Estilo parental permisivo. Mide la tendencia parental hacia la omisión de prácticas disciplinarias o a su aplicación inconsistente. Los ítems provienen de otros instrumentos (Arnold, O'Leary, Wolff, \& Acker, 1993; Robinson, Mandelco, Frost, \& Hart, 1995). La escala consta de seis ítems (confiabilidad $\alpha=.57$, rango: $1-4, M=1.94$, $D E=.56)$. Adicionalmente se obtuvo información acerca de la cantidad de niños menores de 12 años viviendo en el hogar.

\section{Procedimiento}

Los hogares de la muestra nacional con hijos adolescentes fueron visitados de nuevo para encuestar al hijo del beneficiario principal encuestado en la primera encuesta. Las entrevistas se realizaron en el hogar del entrevistado, previo permiso y consentimiento informado del progenitor. Los participantes contestaron individualmente los cuestionarios en un lugar apartado de su hogar. A los respondientes se les informó que la encuesta tenía el propósito de conocer mejor algunos aspectos de su vida y que su participación era voluntaria e independiente de su adscripción al programa. También se les aseguró la confidencialidad de sus datos.

\section{Análisis de datos}

Con el propósito de determinar la existencia de diferencias en el comportamiento prosocial por género y tipo de urbanización se realizaron pruebas $t$ para muestras independientes. Adicionalmente se realizó un modelamiento de ecuaciones estructurales con el objetivo de identificar los predictores más significativos del comportamiento prosocial en adolescentes.

\section{Resultados}

Los resultados de las pruebas $t$ de los puntajes del comportamiento prosocial por género y urbanización mostraron que los puntajes de los hombres $(n=610, M=2.94$, $D E=.64)$ y de las mujeres $(n=483, M=3.11$, $D E=.60)$ difieren significativamente en favor de las mujeres: $t_{(1091)}=-4.64, p<.001$, $d$ de Cohen: .27. Sin embargo, los puntajes de conducta prosocial de jóvenes rurales $(n=426, M=2.97, D E=.63)$ y urbanos $(n=$ $667, M=3.04, D E=.63$ ) no se diferenciaron significativamente: $t_{(1091)}=-1.82, \mathrm{p}=.07, d$ de Cohen: .11.

Se propuso un modelo para explicar la conducta prosocial que se sometió a prueba a través de modelamiento de ecuaciones estructurales. El modelo propuesto incluyó como predictores una variable latente denominada Socialización, la cual estuvo conformada por las escalas que miden competencia social, relaciones positivas con los pares, apoyo social y apoyo de amigos. También se probaron otras variables como estilo parental permisivo, oposicionismo, soledad y religiosidad; así como el número de niños menores de 12 años en el hogar.

El modelo resultante mostró que la variable latente Socialización (competencia social, relaciones positivas con los pares, apoyo social y apoyo de amigos) fue la principal predictora de la conducta prosocial de los adolescentes y que la predice en forma positiva. Es decir, puntajes más altos de conducta prosocial se corresponden con niveles más altos de la variable latente Socialización. Adicionalmente el estilo parental permisivo y la soledad fueron predictores positivos de la conducta prosocial. Es decir, a mayor nivel autoreportado de estilo parental permisivo y de soledad, mayor nivel de conducta prosocial. En contraposición, el oposicionismo y la cantidad de niños menores de 12 años en el hogar la predijeron en forma negativa. Es decir, puntajes más altos de conducta prosocial dependen de puntajes más bajos de oposicionismo y de menor convivencia con niños menores de 12 años en su hogar.

Además de los predictores directos de la conducta prosocial, se observaron efectos indirectos debidos a las interrelaciones entre las variables: Religiosidad influye la conducta prosocial de manera indirecta a 
través de su correlación positiva con Socialización. Es decir, mayor religiosidad concurre con mayores niveles de la variable latente Socialización, que es un predictor positivo de conducta prosocial. Adicionalmente a su influencia directa, oposicionismo, influye la conducta prosocial, también de manera indirecta a través de su correlación positiva con estilo parental permisivo, soledad y número de menores de 12 años en el hogar; éstos son predictores negativos de la conducta prosocial, así como a través de su correlación negativa con Socialización, que es su predictor positivo. Es decir, aquellos con niveles más altos de oposicionismo tienden a reportar niveles más altos de estilo parental permisivo, soledad y convivencia con menores y niveles más bajos de Socialización, todos los cuales concurren con niveles más bajos de conducta prosocial.

También se observó una correlación positiva entre estilo parental permisivo y soledad; es decir, niveles más altos de estilo parental permisivo se relacionan con mayor soledad. Así como una correlación negativa entre oposicionismo y religiosidad, indica que a mayor oposicionismo, menos religiosidad. El modelo probado obtuvo un ajuste adecuado $\left(\chi^{2}(29)=95.82, p<.001\right.$, $\mathrm{CFI}=.977, \mathrm{TLI}=.956, \mathrm{RMSEA}=.046$, $p$ close $=.729)$ y explicó el $61 \%$ de la varianza total de la conducta prosocial. En la Figura 1 se muestran las interrelaciones entre las variables.

\section{Discusión}

El presente estudio tuvo como objetivos identificar predictores de la conducta prosocial de adolescentes mexicanos que viven en condiciones de pobreza y determinar diferencias de género y por urbanización en el comportamiento prosocial de estos jóvenes. No se observaron diferencias por tipo de localidad de residencia. Sin embargo, los datos apoyaron las hipótesis del estudio ya que se observaron diferencias de género en el comportamiento prosocial de los adoles- centes, y el modelamiento de ecuaciones estructurales confirmó la importancia relativa de los indicadores del comportamiento social, agrupados en la variable latente Socialización (relaciones positivas con los pares, competencia social, apoyo social y apoyo de amigos), en la predicción de la conducta prosocial. De hecho, estos indicadores constituyeron el predictor más importante de la conducta prosocial, mientras que los otros indicadores contribuyeron en menor medida a su explicación.

Las diferencias de género en comportamiento prosocial en favor de las mujeres son consistentes con las encontradas por Brittian y Humphries (2015) y difieren de la ausencia de diferencias reportadas por Guevara et al. (2015) y de las diferencias a favor de los varones reportadas por Lai et al. (2015). Cabe acotar que el tamaño del efecto de este resultado sugiere que estas diferencias son pequeñas, lo que explica parcialmente la inconsistencia de los resultados previamente reportados. Al ser ésta una muestra grande, permite la identificación de efectos pequeños. Por otra parte, los datos mostraron que los niveles de comportamiento prosocial son similares en los jóvenes que viven en ambientes rurales y urbanos.

El resultado en cuanto a que los mejores predictores de la conducta prosocial son el comportamiento social positivo y el apoyo percibido confirma la estrecha relación entre las relaciones interpersonales, el apoyo social y la conducta prosocial, en una especie de intercambio social, en el que quienes son socialmente más eficientes y exitosos y más apoyados, tienden a retribuir con mayor altruismo hacia sus pares. Los resultados obtenidos son afines a otros que subrayan la relación positiva entre el comportamiento prosocial y la popularidad y aceptación entre pares (Closson \& Hymel, 2016; JensenCampbell et al., 2010; Laninga-Wijnen et al., 2016; Ruschoff et al., 2015); y entre la conducta prosocial y el apoyo de otros (Plenty et al., 2015). Conforme también con esta relación sinérgica positiva, el comportamiento social eficiente es un predictor de variables relevantes como las expectativas 
laborales positivas (Palomar \& Victorio, 2016).

También coherente con la relación negativa entre conducta hostil y comportamiento prosocial reportada previamente (Lai et al., 2015), menor oposicionismo se relacionó con mayor altruismo. En contraposición, la relación positiva entre soledad y comportamiento prosocial es intrigante, ya que indica que la tendencia hacia el aislamiento social promueve el comportamiento altruista. Esto puede estar relacionado con esfuerzos activos de los jóvenes que se sienten aislados a acercarse a los demás a través de comportamientos altruistas, en consonancia con otros resultados que han evidenciado que la conducta prosocial puede ser una estrategia para congraciarse con otros (Kauten \& Barry, 2016) y que tiene un efecto protector contra el rechazo social (Wang et al., 2015). Cabe también la posibilidad de que los jóvenes que se sienten aislados sientan también mayor empatía y compasión, que expresan en comportamientos altruistas (Eberly-Lewis \& Coetzee1, 2015; Padilla-Walker et al., 2015).

Al respecto se ha encontrado que el altruismo está relacionado con valores de responsabilidad social como la compasión, la solidaridad y la vinculación con los demás (Wray-Lake et al., 2016); y que existe una interrelación entre compasión y conducta prosocial a lo largo de la adolescencia (Carlo et al., 2015). De tal manera, la empatía promueve la conducta prosocial (Mesurado \& Richaud, 2016). Mayor investigación podría dilucidar si la empatía media la relación entre soledad y comportamiento prosocial o si el comportamiento prosocial es una estrategia social de vinculación de quienes se sienten aislados. Por ejemplo, la empatía se relaciona con comportamientos de mayor habilidad social (Oros \& Fontana, 2015).

Otro resultado intrigante es el efecto negativo de la cantidad de niños menores de 12 años. Este resultado sugiere que la convivencia con niños pequeños no impulsa el comportamiento prosocial de los adolescentes. Cabe mencionar que la investigación sobre la conducta prosocial señala consis- tentemente que ésta se mantiene en niveles relativamente bajos en la niñez y aumenta sólo hasta la adolescencia tardía (Carlo et al., 2015; Lai et al., 2015; Malti et al., 2015; Wray-Lake et al., 2016). Una explicación posible del resultado obtenido es que los niños menores muestran menos conducta prosocial en casa debido a su edad y que esto permea el comportamiento de los adolescentes, al transmitir el mensaje de que menos altruismo es la normativa. La correlación positiva entre número de menores de 12 años en el hogar y oposicionismo refuerza esta suposición, ya que sugiere que la influencia de los menores está más orientada hacia el comportamiento antagónico.

Otros predictores revelan una relación más difícil de explicar, como el que un estilo parental más permisivo sea precursor de la conducta prosocial. Este resultado sugiere que menos control parental disciplinario promueve comportamientos en favor de los demás; lo cual es contrario a otros resultados que subrayan el impacto negativo de las prácticas parentales permisivas (Chaparro \& Grusec, 2016) en el comportamiento prosocial de los hijos. La relación positiva entre estilo parental permisivo y oposicionismo sugiere que este estilo auspicia el comportamiento antagónico; ésto hace improbable que la relación positiva entre comportamiento prosocial y estilo permisivo, se deba a que este último propicie un clima social positivo proclive a la conducta prosocial.

También contrario a lo reportado en la literatura (Brittian \& Humphries, 2015; Li \& Chow, 2015; Saroglou et al., 2005), los datos no apoyan una relación directa entre la religiosidad y la conducta prosocial, aunque sí se observó una relación indirecta debida a la covariación de la religiosidad con comportamientos sociales efectivos y positivos. Es decir, los más religiosos tienden a ser más hábiles socialmente, con más relaciones interpersonales positivas, reciben más apoyo y son menos hostiles, y, por lo tanto, quienes tienen estas características son más altruistas.

Además de su influencia directa sobre la conducta prosocial, tanto oposicionismo 
como estilo parental permisivo mostraron también efectos indirectos por su correlación con predictores directos de la conducta prosocial. Tal es el caso de oposicionismo con Socialización, soledad y número de menores de 12 años, y de estilo parental permisivo con soledad y oposicionismo. Es conveniente puntualizar que, si bien existe una relación positiva considerable $(r=.45)$ entre soledad y oposicionismo, que indica que los adolescentes que tienden a aislarse también tienden a mostrar mayor antagonismo, el oposicionismo es un predictor negativo de la conducta prosocial, mientras que soledad es un predictor positivo.

Los jóvenes que crecen en ambientes de pobreza constituyen una población vulnerable que enfrenta no sólo carencias materiales, sino también las desventajas sociales y educativas características de la desigualdad social. La conducta prosocial puede ser instrumental en la acotación de los efectos de la pobreza extrema porque al coadyuvar a aumentar el capital social disponible para la comunidad, aumentan también las posibilidades de progreso de ésta. Es este comportamiento social relevante para el futuro de una población poco estudiada.

El presente estudio tiene como fortaleza principal que los resultados obtenidos provienen de una muestra nacional probabilística de hogares que viven en condiciones de pobreza, lo que genera confianza para extrapolar los datos a los adolescentes mexicanos que viven en condiciones similares. También el tamaño de la muestra es una fortaleza, ya que al ser grande permite reducir el sesgo del error estándar. Sin embargo, el estudio tiene algunas debilidades como el diseño transversal de la encuesta, que imposibilita determinar relaciones causales entre las variables. Además los datos provienen de autoreportes, lo que no permite excluir sesgos de memoria o de deseabilidad social, por lo que es conveniente tomar en consideración estas características del estudio para interpretar sus resultados. A pesar de esta precaución, consideramos que es posible utilizar los presentes resultados en la planeación de estrategias de intervención con adolescentes, con el propósito de incentivar la conducta prosocial en las generaciones futuras.

En conclusión, los resultados mostraron que puntajes más altos en Socialización, estilo parental permisivo y soledad, así como puntajes más bajos en oposicionismo y menor número de niños menores de 12 años en el hogar predijeron puntajes más altos en conducta prosocial. Los resultados son congruentes con la noción general de que un ambiente de compasión, solidaridad, vinculación y confianza es importante en la promoción del desarrollo de valores de responsabilidad social (Wray-Lake et al., 2016). Posibles investigaciones futuras podrían dilucidar si estos predictores difieren en poblaciones de otro nivel socioeconómico e identificar predictores específicos de la conducta prosocial para poblaciones provenientes de distintos estratos sociales. 


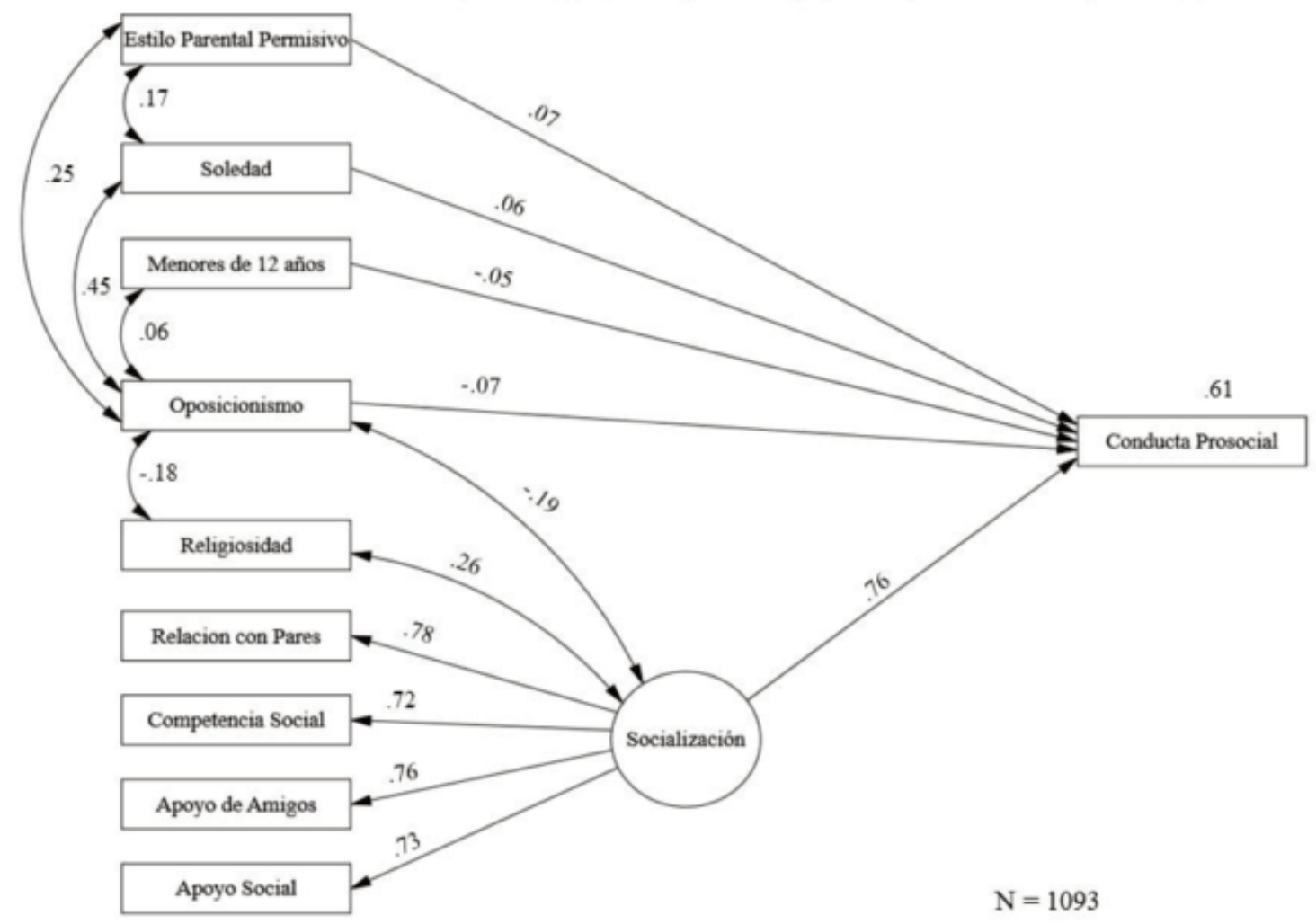

Figura 1. Determinantes de la conducta prosocial en adolescentes

\section{Referencias bibliográficas}

Allgaier, K., Zettler, I., Wagner, W., Püttmann, S., \& Trautwein, U. (2015). Honesty-humility in school: Exploring main and interaction effects on secondary school students' antisocial and prosocial behavior. Learning and Individual Differences, 43, 211-217. http://dx.doi.org/10.1016/j.lindif.2015.08.005. Arnold, D.S., O'Leary, S.G., Wolff, L.S., \& Acker, M. M. (1993). The Parenting Scale: A measure of dysfunctional parenting in discipline situations. Psychological Assessment, 5, 137-144. http://dx.doi. org/ 10.1037 /1040-3590.5.2.137.

Barry, C.T., Lui, J.H.L., \& Anderson, A.C. (2017). Adolescent narcissism, aggression, and prosocial behavior: The relevance of socially desirable responding. Journal of Personality Assessment, 99, 46-55. http://dx. doi.org/10.1080/00223891.2016.1193812.

Brittian, A.S. \& Humphries, M.L. (2015). Prosocial behavior during adolescence. En James D. Wright (Ed.), International encyclopedia of the social \& behavioral sciences (2nd ed., Vol. 19, pp. 221-227). Oxford: Elsevier.

Burnett Heyes, S., Jih, Y.-R., Block, P., Hiu, C.F., Holmes, E. A., \& Lau, J. Y. F. (2015). Relationship reciprocation modulates resource allocation in adolescent social networks: Developmental effects. Child Development, 86, 1489-1506. http://dx.doi.org/10.1111/ cdev. 12396 
Caprara, G. \& Pastorelli, C. (1993). Early emotional instability, prosocial behavior, and aggression: some methodological aspects. European Journal of Personality, 7, 19-36. http://dx.doi.org/10.1002/per.2410070103.

Carlo, G., Crockett, L.J., Randall, B.A., \& Roesch, S.C. (2007). A latent growth curve analysis of prosocial behavior among rural adolescents. Journal of Research on Adolescence, 17, 301-324. http://dx.doi.org/10. 1111/j.1532-7795.2007.00524.x.

Carlo, G., Crockett, L.J., Wilkinson, J.L., \& Beal, S.J. (2011). The Longitudinal relationships between rural adolescents' prosocial behaviors and young adult substance use. Journal of Youth and Adolescence, 40, 1192-1202. http://dx.doi.org/10.1007/s109 64-010-9588-4.

Carlo, G., Padilla-Walker, L.M., \& Nielson, M.G. (2015). Longitudinal bidirectional relations between adolescents' sympathy and prosocial behavior. Developmental Psychology, 51, 1771-1777. http://dx.doi.org /10.1037/dev0000056.

Chaparro, M.P. \& Grusec, J.E. (2016). Neuroticism moderates the relation between parenting and empathy and between empathy and prosocial behavior. MerrillPalmer Quarterly, 62, 105-128. http://dx. doi.org/10.13110/merrpalmquar1982 .62.2.0105.

Closson, L.M. \& Hymel, S. (2016). Status differences in target-specific prosocial behavior and aggression. Journal of Youth and Adolescence, 45, 1836-1848. http://dx.doi. org/10.1007/s10964-016-0481-7.

Davis, M.H. (1980). A multidimensional approach to individual differences in empathy. JSAS Catalog of Selected Documents in Psychology, 10, 85. Recuperado de http:// www.uv.es/ friasnav/Davis 1980.pdf.

De Jong Gierveld, J. \& van Tilburg, T. (1999). Manual of the Loneliness Scale. Amsterdam: VU University Amsterdam, Department of Social Research Methodology. Recuperado de http://home.fsw. vu.nl/TG. van. Tilburg/manual_loneliness_scale_19 99.html.
DuBois, D.L., Felner, R., Brand, S., \& Lease, A.M. (1996). Early adolescent self-esteem: A developmental-ecological framework and assessment strategy. Journal of Research on Adolescence, 6, 543-579.

Eberly-Lewis M.B. \& Coetzee1, T.M. (2015). Dimensionality in adolescent prosocial tendencies: Individual differences in serving others versus serving the self. Personality and Individual Differences, 82, 1-6. http:// dx.doi.org/10.1016/j.paid.2015.02.032.

Flynn, E., Ehrenreich, S.E., Beron, K.J., \& Un derwood, M.K. (2015). Prosocial behavior: Long-term trajectories and psychosocial outcomes. Social Development, 24, 462482. http://dx.doi.org/10.1111/sode. 12100.

Gresham, F.M. \& Elliot, S.N. (1990). Social skills rating system manual. Circle Pines, MN: American Guidance Service.

Grulke, N., Bailer, H., Blazer, G., Geyer, M., Bräler, E., \& Abani, C. (2003). Measuring religious attitudes: reliability and validity of the German version of the Systems of Belief Inventory (SBI-15D-D) in a representative sample. Mental Health, Religion and Culture, 6, 203-213. http://dx.doi.org/10.1080/ 1367467031000087665.

Guevara, I.P., Cabrera, V.E., Gonzalez, M.R., \& Devis, J.V. (2015). Empathy and sympathy as mediators between parental inductive discipline and prosocial behavior in Colombian families. International Journal of Psychological Research, 8, 34-48. Recuperado de http://www.scielo.org.co/scielo. php?script $=$ sci_arttext\&pid=S2011-208420 $15000200004 \& \operatorname{lng}=$ en\&tlng=en.

Harper, J.M., Padilla-Walker, L.M., \& Jensen, A.C. (2016). Do siblings matter independent of both parents and friends? Sympathy as a mediator between sibling relationship quality and adolescent outcomes. Journal of Research on Adolescence, 26, 101-114. http://dx.doi.org/10.1111/jora.12174.

Jensen-Campbell, L.A., Knack, J.M., \& Gomez, H.L. (2010). The psychology of nice people. Social and Personality Psycho- 
logy Compass, 4, 1042-1056. http://dx.doi. org/10.1111/j.1751-9004.2010.00307.x.

Kauten, R.L. \& Barry, C.T. (2016). Adolescent narcissism and its association with different indices of prosocial behavior. Journal of Research in Personality, 60, 36-45. http: //dx.doi.org/10.1016/j.jrp.2015.11.004.

Koenig, H., Pargament, K., Perez, L., \& Smith, B. (1998). Patterns of positive and negative religious coping with major life stressors. Journal for the Scientific Study of Religion, 37, 710-724. http://dx.doi.org/10.2307/138 8152 .

Lai, F.H.Y., Siu, A.M.H., \& Shek, D.T.L. (2015). Individual and social predictors of prosocial behavior among Chinese adolescents in Hong Kong. Frontiers in Pediatrics, 3, 39. http://dx.doi.org/10.3389/fped.2015. 00039.

Laninga-Wijnen, L., Harakeh, Z., Dijkstra, J. K., Veenstra, R., \& Vollebergh, W. (2016). Aggressive and prosocial peer norms. Change, stability, and associations with adolescent aggressive and prosocial behavior development. The Journal of Early Adolescence. http://dx.doi.org/10.1177/027243 1616665211

Li, K.K. \& Chow, W.Y. (2015). Religio sity/spirituality and prosocial behaviors among Chinese Christian adolescents: The mediating role of values and gratitude. Psychology of Religion and Spirituality, 7, 150-161. http://dx.doi.org/10. 1037/a00 38294.

Ma, H.K., Cheung, P.C., \& Shek, D.T.L. (2007). The relation of prosocial orientation to peer interactions, family social environment and personality of Chinese adolescents. International Journal of Behavioral Development, 31, 12-18 . http://dx.doi.org/ 10.1177/0165025406073504.

Malti, T., Averdijk, M., Zuffianò, A., Ribeaud, D., Betts, L.R., Rotenberg, K.J., \& Eisner, M. P. (2015). Children's trust and the development of prosocial behavior. International Journal of Behavioral Development, 40, 262-270. http://dx.doi.org/10.1177/016502 5415584628 .
Mesurado, B. \& Richaud, M.C. (2016). The relationship between parental variables, empathy and prosocial-flow with prosocial behavior toward strangers, friends, and family. Journal of Happiness Studies. http:// dx.doi.org/10.1007/s10902-016-9748-7.

Oros, L.B. \& Fontana Nalesso, A.C. (2015). Niños socialmente hábiles: ¿Cuánto influyen la empatía y las emociones positivas? Interdisciplinaria, 32, 109-125.

Padilla-Walker, L.M., Carlo, G., Christensen, K.J., \& Yorgason, J.B. (2012). Bidi rectional relations between authoritative parenting and adolescents' prosocial behaviors. Journal of Research on Adolescence, 22, 400408. http://dx.doi.org/10.1111/ j.1532-77 95.2012.00807.x.

Padilla-Walker, L.M., Fraser, A.M., Black, B.B., \& Bean, R.A. (2015). Associations between friendship, sympathy, and prosocial behavior toward friends. Journal of Rese arch on Adolescence, 25, 28-35. http://dx. doi.org/10.1111/jora.12108.

Palomar Lever, J. (2015). Rendimiento académico, ajuste psicosocial y bienestar subjetivo en adolescentes hijos de beneficiarios del programa de desarrollo humano Oportunidades. México: Universidad Iberoamericana.

Palomar Lever, J. \& Victorio Estrada, A. (2016) Expectativas laborales en la adolescencia: Correlatos psicosociales. Interdisciplinaria, 33(1), 95-110. https://doi.org/10.16888/interd.2016.33.1.6

Pastorelli, C., Lansford, J.E., Luengo Kanacri, B.P., Malone, P. S., Di Giunta, L., Bacchini, D. \& Sorbring, E. (2016). Positive parenting and children's prosocial behavior in eight countries. Journal of Child Psychology and Psychiatry, 57, 824-834. http://dx.doi.org/ 10.1111/jcpp.12477.

Pelham, W.E., Gnagy, E.M., Greenslade, K.E., \& Milich, R. (1992). Teacher ratings of DSM-III-R symptoms for the disruptive behavior disorders. Journal of the American Academy of Child and Adolescent Psychiatry, 31, 210-218. http://dx.doi.org/10.1 097/00004583-199203000-00006. 
Plenty, S., Östberg, V., \& Modin, B. (2015). The role of psychosocial school conditions in adolescent prosocial behavior. School Psychology International, 36, 283-300. http: //dx.doi.org/10.1177/0143034315573350.

Robinson, C.C., Mandelco, B., Frost, O.S., \& Hart, C.H. (1995). Authoritative, authoritarian and permissive parenting practices: Development of a new measure. Psychological Reports, 77, 819-830. http://dx.doi. org/10.2466/pr0.1995.77.3.819.

Ruschoff, B., Dijkstraa, J.K., Veenstra, R., \& Lindenberg, S. (2015). Peer status beyond adolescence: Types and behavioral associations. Journal of Adolescence, 45, 1-10. http://dx.doi.org/10.1016/j.adolescence. 2015.08. 013.

Saroglou, V., Pichon, I., Trompette, L., Vers chueren, M., \& Dernelle, R. (2005). Prosocial behavior and religion: New evidence based on projective measures and peer ratings. Journal for the Scientific Study of Religion, 44, 323-348. http://dx.doi.org /10.1111/j.1468-5906.2005.00289.x.

van Hoorn, J., van Dijk, E., Meuwese, R., Rieffe, C., \& Crone, E.A. (2016). Peer influence on prosocial behavior in adolescence. Journal of Research on Adolescence,
26, 90-100. http://dx.doi.org/10.1111/jora. 12173

van Rijsewijk, L., Dijkstra, J.K., Pattiselanno, K., Steglich, C., \& Veenstra, R. (2016). Who helps whom? Investigating the development of adolescent prosocial relationships. Developmental Psychology, 52, 894-908. http://dx.doi.org/10.1037/dev0000106.

Wang, S., Zhang, W., Li, D., Yu, C., Zhen, S., \& Huang, S. (2015). Forms of aggression, peer relationships, and relational victimization among Chinese adolescent girls and boys: roles of prosocial behavior. Frontiers in Psychology, 6, 1264. http://dx.doi.org/10. 3389/fpsyg.2015.01264.

Wray-Lake, L., Syvertsen, A. K., \& Flanagan, C. A. (2016). Developmental change in social responsibility during adolescence: An ecological perspective. Developmental Psychology, 52, 130-142. http://dx.doi.org/10.1037/dev 0000067.

Zimet, G., Dahlem, N., Zimet, Z., \& Farley, G. (1988). The Multidimensional Scale of Perceived Social Support. Journal of Personality Assessment, 52, 30-41. http://dx. doi.org/10.1207/s15327752jpa5201__. 
